ERRATUM

\title{
Metabolically constrained regulatory networks
}

\section{Hannah Stower}

Nature Reviews Genetics 15, 65 (2014)

In this In Brief, the incorrect journal was listed in the citation. The citation should have been: Chandrasekaran, S. \& Price, N. D.

Metabolic constraint-based refinement of transcriptional regulatory networks. PLoS Comput. Biol. 9, e1003370 (2013).

The article has been corrected online. The editors apologize for this error. 